\title{
Towards Sophisticated Control of Robotic Manipulators: An Experimental Study on a Pseudo-Industrial Arm
}

\author{
Jamshed Iqbal1,2,* - Muhammad Imran Ullah² - Abdul Attayyab Khan ${ }^{3}$ - Muhammad Irfan 4 \\ 1 National University of Computer and Emerging Sciences, Islamabad, Department of Electrical Engineering, Pakistan \\ ${ }^{2}$ COMSATS Institute of Information Technology, Islamabad, Department of Electrical Engineering, Pakistan \\ 3 University of Genova, DIBRIS, Italy \\ ${ }_{4}^{4}$ University of Engineering and Technology, Taxila, Department of Electrical Engineering, Pakistan
}

Robotic manipulators have reshaped industrial processes. The scientific community has witnessed an ever increasing trend in robots deployed to accomplish various tasks in industry. The complex nature and constrained requirements of robots may demand non-trivial control approaches. This paper deals with the design, simulation and hardware realization of two sophisticated control strategies: computed torque control (CTC) and variable structure control (VSC) on a pseudo-industrial manipulator with six degrees of freedom (DOF). Based on the derived kinematic and dynamic models of the robot, control laws have been formulated, which are then subjected to various test inputs in a simulation to characterize the tracking performance. The simulation results were then validated by implementing control laws on a custom-developed pseudo-industrial autonomous articulated robotic educational platform (AUTAREP). The experimental results show the effectiveness of the control strategies to track a desired trajectory.

Keywords: robot control, manipulator, robust laws, industrial robots

Highlights

- $\quad$ This paper presents advanced strategies to control a highly non-linear system like a multiple Degree Of Freedom (DOF) robotic arm.

- $\quad$ The strategies include computed torque control (CTC) and variable structure control (VSC).

- Design parameters using both strategies have been investigated in a simulation.

- $\quad$ The strategies were carried out on a custom-developed autonomous articulated robotic educational platform (AUTAREP).

- $\quad$ Trajectory tracking results showed that the derived laws can effectively track the desired reference input for both strategies.

\section{INTRODUCTION}

Today, robots are being deployed to accomplish tasks having strict requirements of accuracy, precision, repeatability, mass production and quality. A major breakthrough was reported with the advent of feedback control systems and self-correcting mechanisms. The development of multiple degree of freedom (multi-DOF) manipulators has contributed significantly to modern robots. Industrial robots are primarily multi-DOF anthropomorphic manipulators. The past few years have seen a large rise in the use of industrial robots and this trend will likely continue, as highlighted in [1].

The performance of a robotic manipulator is characterized by a well-defined control approach [2] Classical or trivial control strategies are usually based on linear control laws while modern approaches are nonlinear in nature. The approach to controlling a multi-DOF manipulator must be robust enough to cope with the effects of inherent nonlinearities and coupling in the robot dynamics [3] to [5]. Classical approaches suffer from various issues, which can be avoided by merging these with advanced control strategies or using an advanced strategy in standalone.

Control of multi-DOF robotic manipulators is a vital research area today. However, most of the reported research is either limited to the implementation of linear control approaches or the simulation of sophisticated control strategies. In contrast, the present work investigates advanced approaches like computed torque control (CTC) and variable structure control (VSC) from a simulation viewpoint as well as its physical realization on a custom-developed autonomous articulated robotic educational platform (AUTAREP).

\section{CONTROL DESIGN}

The study of manipulators for diversified applications has highlighted the need for sophisticated algorithms for their control and trajectory planning. The objective in the design of robotic manipulators is to control both the position and the orientation of the tool in a 3D workspace.

The scientific community has reported both classical and robust strategies for robot control. 
With regard to classical approaches, Iqbal et al. have proposed proportional integral derivative (PID) controllers for mobile robots [6] and multi-DOF serial link robotic exoskeletons [7] and [8]. The role of PID control in industrial automation has been presented in [9], which formulates a nonlinear PID control law to ensure global asymptotic stability (GAS). Classical approaches, when combined with modern control strategies, improve transient response in uncertain scenarios as highlighted in [10]. Combining VSC with PID and adaptive control strategies, Jingmei et al. have improved precision in trajectory tracking of a robotic manipulator [11]. Here, chattering has been reduced via increased system response time. Tahir and Jaimoukha have proposed a model predictive robust controller [12] for linear discrete-time systems subjected to polytopic constraints and bounded disturbances. The proposed control approach is novel in that the outer controller incorporates a state-feedback structure where feedback gains are considered as decision variables in online optimization [13].

PID has been the main workhorse in the industrial sector. However, researchers have recently shown an active interest in the development and applications of nonlinear control methodologies applied to robotic manipulators. A comprehensive review of control strategies for manipulators is reported in [3].

The overall control problem consists of kinematic and dynamic modeling, followed by the design of a control law. The kinematics of the AUTAREP manipulator has been derived in [14] using DenavitHartenberg (DH) representation while the system dynamics has been modeled in [15] using Euler Lagrange equation (Eq. (1)).

$$
\tau=\mathbf{M}(\mathbf{q}, \dot{\mathbf{q}}) \ddot{\mathbf{q}}+\mathbf{V}(\mathbf{q}, \dot{\mathbf{q}})+\mathbf{G}(\mathbf{q})+\mathbf{f}(\dot{\mathbf{q}}),
$$

where $\mathbf{M}(\mathbf{q}, \dot{\mathbf{q}})$ is a $4 \times 4$ inertia matrix, $\mathbf{V}(\mathbf{q}, \dot{\mathbf{q}}), \mathbf{G}(\mathbf{q})$ and $\mathbf{f}(\dot{\mathbf{q}})$ are $4 \times 1$ vectors of Coriolis centrifugal force, Gravitational force and Frictional force, respectively. $\tau$ is the $4 \times 1$ torque vector applied to the joints of the robot and $\mathbf{q}, \dot{\mathbf{q}}$ and $\ddot{\mathbf{q}}$ are $4 \times 1$ vectors for angular position, velocity and acceleration respectively.

This paper deals with two modern control strategies namely CTC and VSC.

\subsection{CTC}

CTC is a special type of feedback linearization technique with symmetric, constant and positive definite controller gains. CTC can be utilized effectively in cases where there are known nonlinear dynamic parameters and uncertainties. For the CTC law, the expression for the manipulator system in Eq. (1) can be written as Eq. (2).

$$
\tau=\mathbf{M}(\mathbf{q}, \dot{\mathbf{q}})\left(\ddot{\mathbf{q}}_{\mathbf{d}}-2 \lambda \dot{\mathbf{e}}-\lambda^{2} \mathbf{e}\right)+\mathbf{V}(\mathbf{q}, \dot{\mathbf{q}})+\mathbf{G}(\mathbf{q}),
$$

where the vector $\mathbf{q}=\left[q_{1} q_{2} q_{3} q_{4}\right]^{\mathrm{T}}$ corresponds to the first four joints of the manipulator. $\mathbf{q}_{\mathbf{d}}, \dot{\mathbf{q}}_{\mathbf{d}}$ and $\ddot{\mathbf{q}}_{\mathbf{d}}$ are the desired joint angle position vector

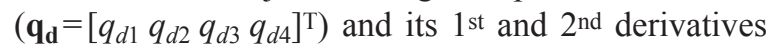
respectively. $\mathbf{e}=\mathbf{q}-\mathbf{q}_{\mathbf{d}}$ is the error signal with $\dot{\mathbf{e}}$ as its $1^{\text {st }}$ derivative. $\tau$ is the required control output and is represented by $\boldsymbol{\tau}=\left[\begin{array}{ll}\tau_{1} & \tau_{2} \tau_{3} \tau_{4}\end{array}\right]^{\mathrm{T}}$. The gain matrix $\lambda=\operatorname{diag}\left\{\lambda_{1} \lambda_{2} \lambda_{3} \lambda_{4}\right\}$ can be used to alter the system dynamics.

\subsection{VSC}

VSC has the potential to eliminate uncertainties and disturbances present in the system. A switching surface is designed and the main task of the controller is to drive the system states to this surface. The system then remains on the switching surface to reduce disturbances and modeling uncertainties. VSC is a robust control strategy using high frequency switching control to alter the dynamics of the nonlinear system. The VSC law for the robotic manipulator, using the sliding manifold $\mathbf{S}=\dot{\mathbf{e}}+\mathbf{C e}$, is given in Eq. (3).

$$
\begin{aligned}
\tau & =\mathbf{M}(\mathbf{q}, \dot{\mathbf{q}})\left(\ddot{\mathbf{q}}_{\mathbf{d}}-\mathbf{C} \dot{\mathbf{e}}\right)+\mathbf{V}(\mathbf{q}, \dot{\mathbf{q}})+\mathbf{G}(\mathbf{q})- \\
& -\mathbf{K} \operatorname{sgn}(\mathbf{C e}+\dot{\mathbf{e}}),
\end{aligned}
$$

where the matrices $\mathbf{C}=\operatorname{diag}\left\{c_{1} c_{2} c_{3} c_{4}\right\} \quad$ and $\mathbf{K}=\operatorname{diag}\left\{k_{1} k_{2} k_{3} k_{4}\right\}$ are the switching gain constant and sliding surface constant, respectively, and can be changed to alter the system dynamics.

\section{SIMULATION}

The controller s-functions were developed in a MATLAB/Simulink based on the derived dynamic model of the manipulator. Various desired trajectories including step, sinusoidal and ramp have been applied to analyze the robustness and effectiveness of the proposed control laws. The overall effect of the plant has been investigated by choosing different values for the system constants, i.e. $\boldsymbol{\lambda}$ for CTC and $\mathbf{C}, \mathbf{K}$ for VSC.

In the case of CTC, varying $\lambda$ revealed that the performance of the system improves by increasing the value of $\boldsymbol{\lambda}$. For example, when considering the second joint (shoulder), a simulated step response is used to investigate the effect of the assigned $\lambda_{2}$ values. Keeping $\lambda_{1}, \lambda_{3}$ and $\lambda_{4}$ constant (unity), the 
step response of the shoulder joint for $\lambda_{2}=1,2,3,4$ is illustrated in Fig. 1a with the corresponding torques plotted in Fig. 1b. No overshoot is observed in any case. However, a significant difference in rise time and settling time is noticeable. When $\lambda_{2}$ is increased from 1 to 4 , the rise time is reduced from $7.13 \mathrm{~s}$ to $1.97 \mathrm{~s}$ while the settling time is reduced from $5.20 \mathrm{~s}$ to $1.86 \mathrm{~s}$ at $\pm 5 \%$ of the desired joint angle. The initial magnitude of torque $(25.9 \mathrm{Nm})$ is required to keep the shoulder joint at its initial position against the gravitational force. The final magnitude of torque is $12.9 \mathrm{Nm}$.
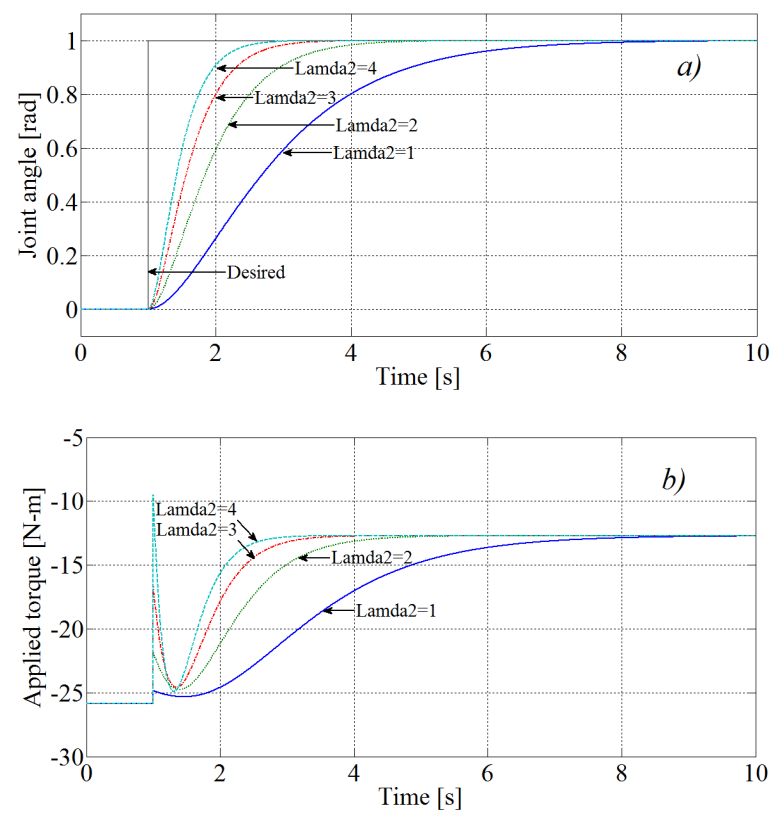

Fig. 1. Shoulder joint a) CTC step response for different values of $\lambda 2$ b) corresponding torques

Where all joints are moving simultaneously, the same value $(\lambda=4)$ has been set for each joint of the manipulator. The corresponding step responses and plots of torque are shown in Fig. 2. With a slight difference in the behaviour of various joints, it is inferred from the results that the various joints exhibit different torque requirements. The shoulder joint requires final torque of $5.4 \mathrm{Nm}$ due to the movement of other joints. The initial and final torque requirements of the base joint are $0 \mathrm{Nm}$ due to the zero gravitational effect. The same requirements in case of the elbow joint are $5.9 \mathrm{Nm}$ and $3.1 \mathrm{Nm}$, respectively. Having few gravitational effects in wrist joint, initial and final torque requirements are $0 \mathrm{Nm}$ and $0.2 \mathrm{Nm}$, respectively.

The equivalent control is designed such that the states of the system are on the sliding manifold, which is defined as $\mathbf{S}=\dot{\mathbf{e}}+\mathbf{C e}$ where $\mathbf{C}$ must satisfy the Hurwitz condition, i.e. $\mathbf{C}>0$. Considering first joint (base) for the sake of brevity, the Lyapunov function, $l_{1}=0.5 s_{1}^{2}$ restricts $k_{1}>0$ The corresponding entries of $\mathbf{C}$ and $\mathbf{K}$ matrices cannot be selected independently without violating the above conditions [16] due to the joints' coupling effects.
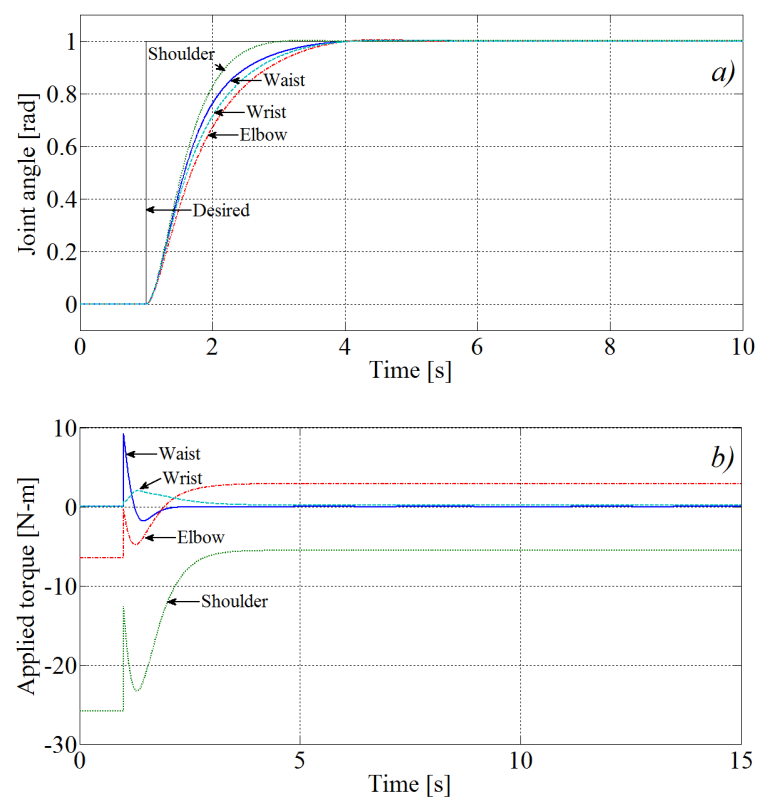

Fig. 2. Joints moving simultaneously, a) CTC step response b) corresponding torques

With $c_{1}=4$, the step response for different values of $k_{1}$ is illustrated in Fig. 3. Comparing the responses, it is clear that the waveform corresponding to $k_{1}=8$ exhibits relatively less rise time and settling time. Thus, the optimum response is achieved with $k_{1}=8$.

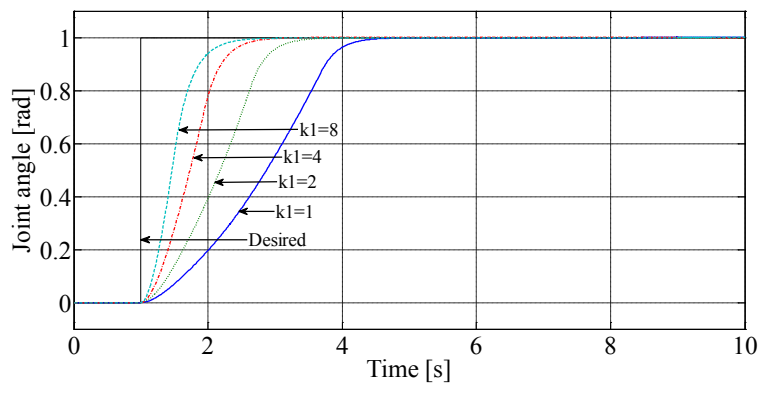

Fig. 3. VSC step response of the base joint for different values of $k_{1}$

With tuned values of the $\mathbf{C}$ and $\mathbf{K}$ matrices, the step response of various joints moving simultaneously is shown in Fig. 4a. It can be inferred from the plot that the elbow joint and wrist joint, even after reaching their desired position, are not stable until the shoulder 
joint reaches its final position and is stabilized. This is in accordance with the coupling effects of the joints. Looking at the applied torque plot shown in Fig. 4b, the switching effect is observable.
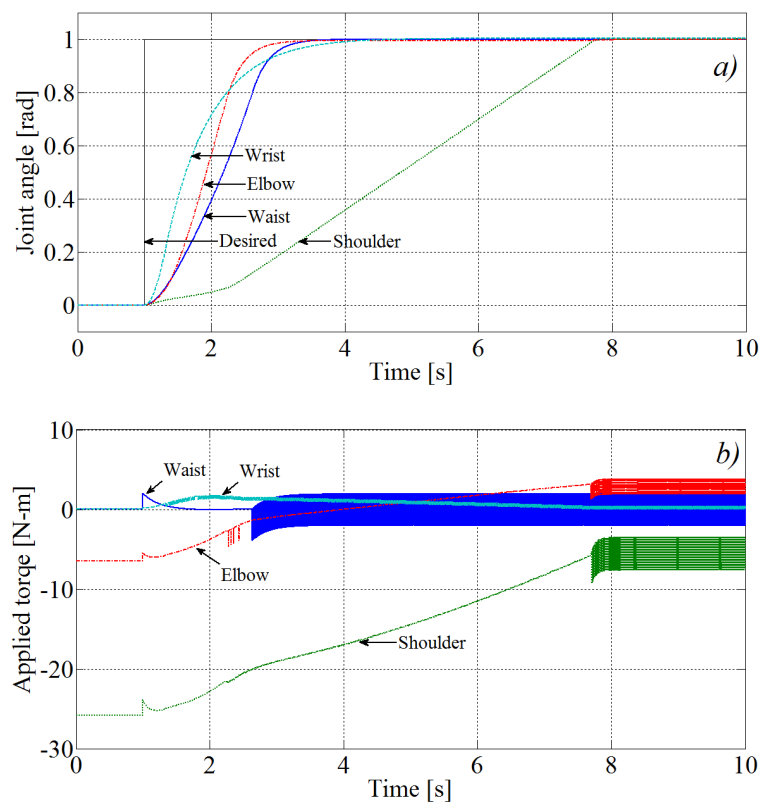

Fig. 4. Joints moving simultaneously

a) VSC step response, b) corresponding torques

\section{EXPERIMENTAL SETUP AND RESULTS}

To validate the simulation results, experiments have been conducted on an indigenously developed AUTAREP illustrated in Fig. 5. It is a mini-industrial open-source, novel and complete robotic system that finds potential in training interns, imparting mechatronics concepts to engineering students and validating advanced algorithms for trajectory generation and control, object manipulation and grasping, path planning, etc. [17].

The mechanical system of the platform is built around a 6-DOF serial robotic manipulator. The arm's geometrical configuration resembles that of the human arm. Six precise DC servo motors actuate the robot while the sensory system is comprised of encoders and force sensing resistors (FSR) in addition to an on-board camera. The primary features of AUTAREP are mentioned in [15]. The designed electronic system mainly consists of an embedded controller DSPIC33F and $6 \mathrm{~A} / 50 \mathrm{~V}$ rated custom-designed motor drivers. The hardware and software architectures of the platform are detailed in [17].

First, the CTC law is implemented on the manipulator. The trajectory tracking performance for different gains $(\lambda \mathrm{i})$ is then observed for base, shoulder, elbow and wrist joints with each joint moved at a time. Fig. 6 illustrates the step responses of the shoulder joint and elbow joint with $20^{\circ}$ set as the desired angle.

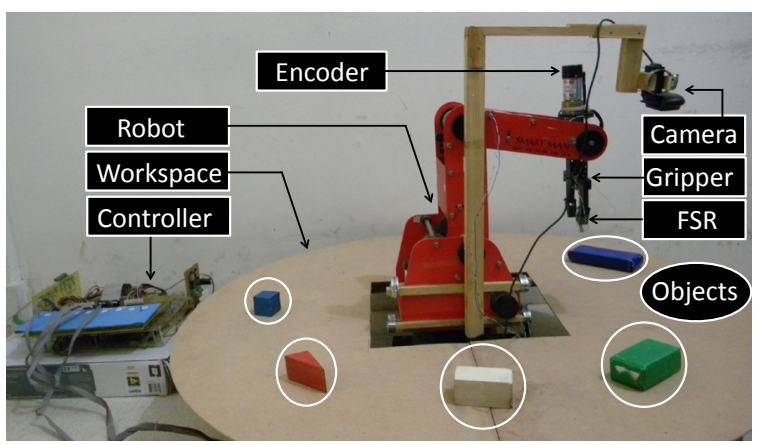

Fig. 5. AUTAREP - A custom-developed pseudo-industrial framework

CTC simulation and hardware implementation both show that the increase in the gain-constant results in better performance by improving the system's response. In contrast to the simulation, the hardware results show that for same value of gain constant, each joint exhibit slightly different response. This is due to the fact that each joint's motor produces a different torque and speed.
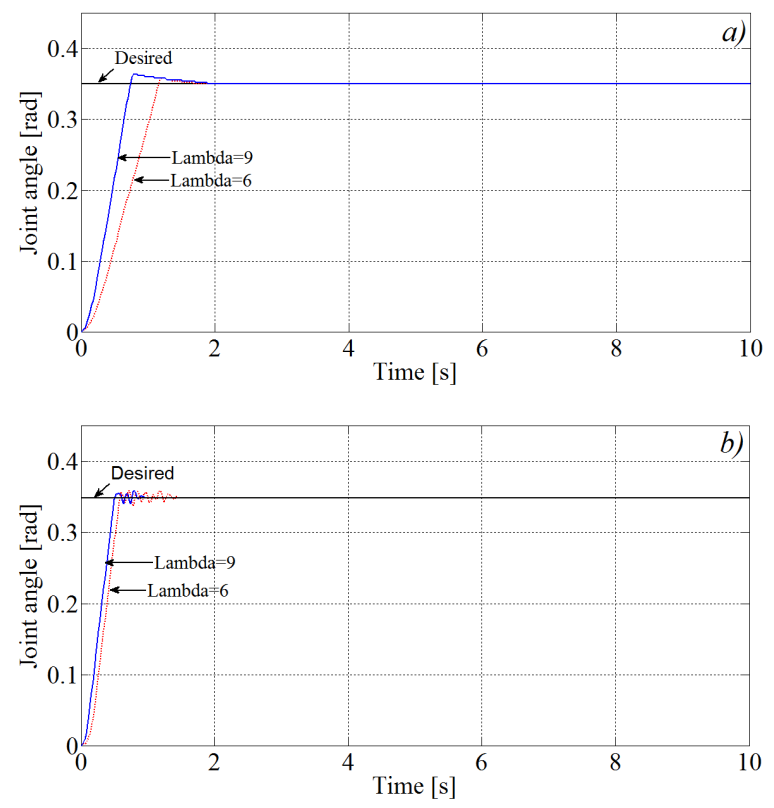

Fig. 6. CTC step response of the a) shoulder joint, b) elbow joint

After simple trajectory tracking experiments, the coupling effect was also studied in order to determine the VSC law on the physical manipulator. Joints were moved simultaneously as well as independently to 
observe this effect. The results are illustrated in Fig. 7. It can be inferred from Fig. 7a that the shoulder joint exhibits overshoot for a longer period of time and settles to the reference angle after settling of the elbow joint. The coupling effect on the shoulder joint causes it to move faster.

VSC simulation and hardware implementation both confirm a strong coupling effect. In contrast to the simulation, the elbow joint in the hardware implementation does not come to rest until the shoulder joint is stabilized at its destination. This is quite expected due to the fact that a link near the fixture or base is less exposed to mechanical stability.
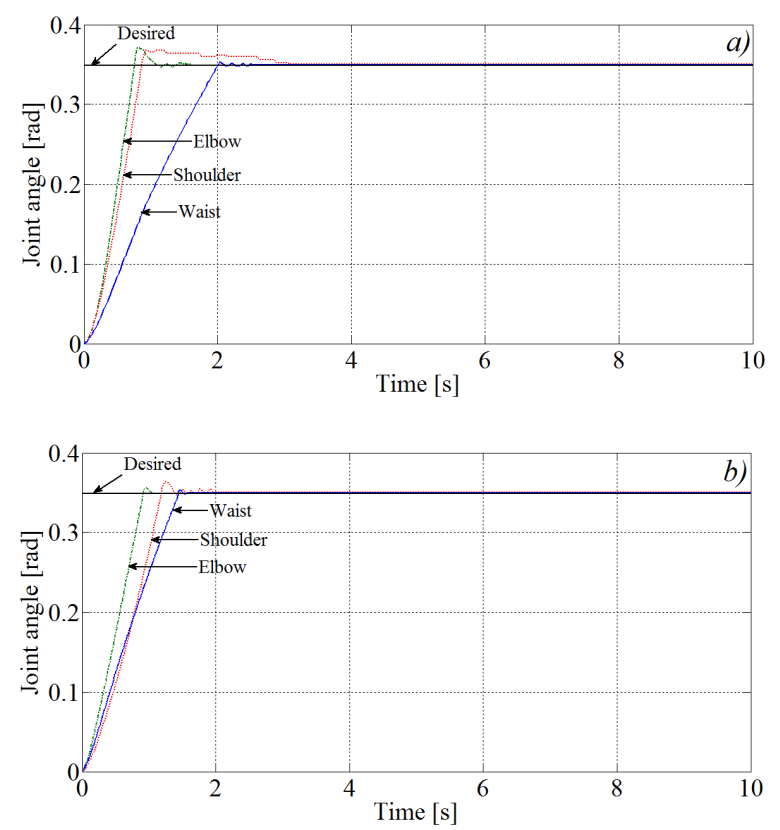

Fig. 7. Step response of base, shoulder and elbow joints when the joints are actuated: a) at the same time, b) one at a time

\section{CONCLUSIONS}

This paper presents the design, simulation and hardware realization of CTC and VSC strategies. The simulation results have been verified through experimental implementation on a physical platform. Trajectory tracking results showed that the derived laws can effectively track the desired reference input for both non-linear control methods. The coupling effects present in the joints are less visible in the simulation but are more prominent in the hardware implementation. Future work will include a task dependent performance comparison of robust control strategies on multi-DOF robotic manipulators.

\section{REFERENCES}

[1] World Robotics (Executive Summary). (2014). International

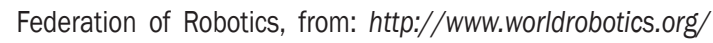
uploads/media/Executive_Summary_WR_2014_02.pdf, accesed at 2015-06-11, p. 11-24.

[2] Caglar, C., Ali, K., Selcuk, M., Sadettin, K., Hakan, Y. (2014). An enhanced control technique for the elimination of residual vibrations in flexible-joint manipulators. Strojniški vestnik Journal of Mechanical Engineering, vol. 60, no. 9, p. 592-599, DOl:10.5545/sv-jme.2014.1698.

[3] Ajwad, S.A., Ullah M.I., Khelifa, B., Iqbal, J. (2014). A comprehensive state-of-the-art on control of industrial articulated robots. Journal of Balkan Tribological Association, vol. 20 , no. 4, p. 499-521.

[4] Siciliano, B., Khatib, 0. (2008). Springer Handbook of Robotics. Springer Science \& Business Media, D0I:10.1007/978-3-54030301-5.

[5] Ajwad, S.A., Iqbal, J., Ullah, M.l., Mehmood, A. (2015). A systematic review of current and emergent manipulator control approaches. Yang, S. (ed.) Frontiers of Mechanical Engineering, Higher Education Press, Beijing and SpringerVerlag Berlin Heidelberg, D0I:10.1007/s11465-015-0335-0.

[6] Iqbal, J., Saad, M.R., Malik, A., Tahir, A.M. (2014). State estimation technique for a planetary robotic rover. Revista Facultad de Ingenieria, vol. 73, p. 58-68.

[7] Iqbal, J., Tsagarakis, N.G., Caldwell, D.G. (2014). Human hand compatible underactuated exoskeleton robotic system. IET Electronic Letters, vol. 50, no. 7, p. 494-496, Dol:10.1049/ el.2014.0508.

[8] Iqbal, J., Khan, A.H., Tsagarakis, N.G., Caldwell, D.G. (2014). A novel exoskeleton robotic system for hand rehabilitation - Conceptualization to prototyping, Biocybernetics and Biomedical Engineering, vol. 34, no. 2, p. 79-89, D0I:10.1016/j.bbe.2014.01.003.

[9] Antonio, Y., Victor, S., Javier, M.V. (2011). Global asymptotic stability of the classical PID controller by considering saturation effects in industrial robots. International Journal of Advance Robotic Systems, vol. 8, p. 34-42, D0l:10.5772/45688.

[10] Kadir, Z.A., Mazlan, S.A., Zamzuri, H., Hudha, K., Amer, N.H. (2015). Adaptive fuzzy-PI control for active front steering system of armored vehicle: Outer loop control design for firing on the move system. Strojniški vestnik - Journal of Mechanical Engineering, vol. 61, no. 3, p. 187-195, D0l:10.5545/svjme.2014.2210.

[11] Jingmei, Z., Haiyang, H., Bo, K. (2012). Studies of adaptive control methods based on VSC for trajectory tracking of robotic manipulators. Proceedings of International Conference on Robotics and Biomimetics, p. 429-434.

[12] Tahir, F., Jaimoukha, I.M. (2013). Robust feedback model predictive control of constrained uncertain systems. Journal of Process Control, vol. 23, no. 2, p. 189-200, D0l:10.1016/j. jprocont.2012.08.003.

[13] Tahir, F., Jaimoukha, I.M. (2013).Causal state-feedback parameterizations in robust model predictive control. Automatica, vol. 49, no. 9, p. 2675-2682, Dol:10.1016/j. automatica.2013.06.015. 
[14] Iqbal, J., Islam, R.U., Khan, A.H. (2012). Modeling and analysis of a 6 DOF robotic arm manipulator. Canadian Journal on Electrical and Electronics Engineering, vol. 3, no. 6, p. 300306.

[15] Manzoor, S., Islam, R.U., Khalid, A., Samad, A., Iqbal, J. (2014). An open-source multi-DOF articulated robotic educational platform for autonomous object manipulation. Robotics and Computer Integrated Manufacturing, vol. 30, no. 3, p. 351362, Dol: 10.1016/j.rcim.2013.11.003.
[16] Shafiei, S.E. (ed.) (2010). Sliding mode control of robot manipulators via intelligent approaches. Advanced Strategies for Robot Manipulators, Intech Open Access Publisher, p. 135172, Dol:10.5772/10193.

[17] Iqbal, U., Samad, A., Nissa, Z., Iqbal, J. (2014). Embedded control system for AUTAREP - a novel autonomous articulated robotic educational platform. Tehnički vjesnik Technical Gazette, vol. 21, no. 6, p. 1255-1261. 